\title{
Altered expression of phosphatase of regenerating liver gene family in non-small cell lung cancer
}

\author{
JUNG-JOO HWANG ${ }^{1,2^{*}}$, SANG-HYUN MIN ${ }^{3 *}$, KI-HYUK SIN ${ }^{1}$, YOUNG-SHIN HEO ${ }^{1}$, \\ KIL-DONG KIM ${ }^{2}$, OOK-JOON YOO ${ }^{1}$ and SEUNG-HYO LEE ${ }^{1}$
}

\begin{abstract}
${ }^{1}$ Graduate School of Medical Science and Engineering, Biomedical Research Center, KAIST Institute for the BioCentury, Korea Advanced Institute of Science and Technology, Daejeon 305-701; ${ }^{2}$ Department of Thoracic and Cardiovascular Surgery, Eulji University Hospital, Daejeon 302-799, Republic of Korea; ${ }^{3}$ Department of

Medicine, Beth Israel Deaconess Medical Center, Harvard Medical School, Boston, MA 02215, USA
\end{abstract}

Received August 1, 2011; Accepted September 16, 2011

DOI: 10.3892/or.2011.1495

\begin{abstract}
Protein tyrosine phophatases (PTPs) are implicated in the tumorigenesis and metastasis of human cancer. The phosphatase of regenerating liver (PRL) gene family, a subgroup of PTPs is also linked to these processes. In many solid cancers, high levels of PRL-3 expression are related with metastasis and poor prognosis. However, the expression patterns of PRL-1 and -2 have not been explored in lung cancer yet. Thus, we investigated the expression levels of PRL-1, -2 and -3 in the tissues of primary lung cancer patients. The protein expression levels of PRL-2, but not PRL-1 and -3 were increased in cancer tissues. However, there was no correlation between mRNA and protein expression levels of the PRLs. Reporter assays showed that PRLs suppressed the activity of the p21 promoter but promoted AP-1 activity. Furthermore, transfection of PRLs showed significantly increased cell proliferation. Therefore, these results suggest that PRL-2 plays an important role in lung cancer and can be a biomarker of lung cancer, substituting for the function of other PRLs.
\end{abstract}

\section{Introduction}

Protein kinases and/or phosphatases participate in signal transduction pathways and modulate phosphorylation levels of cellular proteins that regulate biological functions. One such

Correspondence to: Dr Seung-Hyo Lee, Cellular Immunology Laboratory, Graduate School of Medical Science and Engineering, Biomedical Research Center, KAIST Institute for the BioCentury, Korea Advanced Institute of Science and Technology, Daejeon 305-701, Republic of Korea

E-mail: seung-hyo.lee@kaist.ac.kr

*Contributed equally

Key words: non-small cell lung cancer, PRL-2, p21, AP-1, cell proliferation phosphatase, protein tyrosine phosphatase (PTP), catalyzes the hydrolysis of phosphate monoesters specifically on tyrosine residues (1) and members of the PTP family regulate a variety of cellular processes, especially post-translational modification by participating in every aspect of cellular physiologic and pathogenic process $(1,2)$.

Phosphatase of regenerating liver (PRL) family is a group of PTPs that play a significant role in development and metastasis of various types of cancers (3). Within this family, PRL-1 (PTP4A1), PRL-2 (PTP4A2) and PRL-3 (PTP4A3) share a high degree of similarity. PRLs are overexpressed in a variety of cancer cell lines and tissues such as colorectal cancer (4), prostate cancer (5), breast cancer (6), gastric cancer (7) and liver cancer (8) especially in metastatic cancers. For this reason, PRL family is implicated as a candidate for a biomarker in metastatic and advanced cancers. Among them, PRL-3 is highly expressed in heart and skeletal muscle in normal tissues and low in other tissues. However, PRL-1 and -2 seem to be more ubiquitously expressed than PRL-3 does (9). Thus, it has been thought that PRLs show similar function common to many tissues and biological processes.

In primary lung cancers, there are few studies on the expression pattern of PRLs. In one study, the level of PRL-3 expression was decreased in metastatic primary lung cancer (10), while that of PRL-3 expression was positively correlated with the advanced stage of lung cancer in another study (11). Because of the controversies in primary lung cancer, we investigated the expression patterns and levels of PRL-1, -2 and -3 in primary lung cancer patients. In addition, since PRL-1 and -3 but not PRL-2 have been known to be involved in several oncogenic signaling pathways including PI3K/AKT, C-terminal Src kinase (Csk), Rho GTPases, cyclin-dependent kinase (CDK2), ERK, p53 and p21 (9,12-15), we examined the change of the oncogenic signals by overexpression of PRL-1, -2 and -3 in a cancer cell line.

In this study, we showed that protein expression levels of PRL-2, but not PRL-1 and -3 were increased in cancer tissues but protein levels were not correlated with those of mRNA suggesting that translation of mRNA is regulated by PolyC-RNA-binding protein 1 (PCBP1) like PRL-3 (16) or 
post-translational regulatory mechanism is important. We also showed that PRLs suppressed the activity of p21 promoter and enhanced AP-1 activity with promoter-reporter assay. Finally, overexpression of PRLs through transfection significantly increased cell proliferation. Therefore, these results suggest that PRL-2 may be a biomarker of primary lung cancer and play an important role in the pathogenesis of lung cancer.

\section{Materials and methods}

Sample preparation. Thirty lung cancer and normal samples from non-small cell lung cancer patients were obtained from Eulji University Hospital (Daejeon, Korea). The tumor tissues were confirmed by pathological diagnosis and no distant metastasis was observed by PET-CT scan. None of the 30 cases had received neo-adjuvant radiotherapy or chemotherapy before surgery. The tissues were stored in liquid nitrogen tank until use. Normal tissues were obtained from the remote sites of cancer tissues of the same lobes. This study has been approved by the institutional review board at Eulji University Hospital and informed consent was obtained from participating subjects.

Antibodies and reagents. Goat anti-PRL-1 polyclonal antibody was purchased from Everest Laboratory (Oxfordshire, UK). Chicken anti-PRL-2 polyclonal antibody was purchased from Genway Biotech Inc. (San Diego, CA, USA). Rabbit anti-PRL-3 polyclonal antibody and mouse antibodies against FLAG M2 and $\beta$-actin were obtained from Sigma (St. Louis, MO, USA).

Western blot analysis. Frozen tissue samples (about $100 \mathrm{mg}$ ) were minced with scissors and grinded with small amount of liquid nitrogen using mortar and pestle. Then the tissue samples were transferred to $1.5-\mathrm{ml}$ tube and $300 \mu \mathrm{l}$ of ice-cold RIPA-buffer (phosphate-buffered saline, $\mathrm{pH} 7.4$ containing $1 \%$ NP40 (Sigma) with freshly added PMSF (10 $\mu \mathrm{l} / \mathrm{ml}$ of $10 \mathrm{mg} /$ $\mathrm{ml}$ ) and protease inhibitor cocktail tablet (Roche Diagnostics, Mannheim, Germany) was added. After $1 \mathrm{~h}$ incubation on ice, samples were spun at $10,000 \mathrm{rpm} / \mathrm{min}$ for $10 \mathrm{~min}$ at $4^{\circ} \mathrm{C}$ and supernatants were collected. Two additional centrifugations were performed to produce clarified lysates. Protein concentrations of the resulting lysates were determined by Bio-Rad protein assay (Bio-Rad Laboratories, Hercules, CA, USA). Equivalents of $20 \mu \mathrm{g}$ protein of each sample were denatured at $90^{\circ} \mathrm{C}$ for $5 \mathrm{~min}$. Tissue extracts were detected using antiPRL-1, PRL-2 and PRL-3 antibodies followed by SDS-PAGE and immunoblot analysis. Band intensity of Western blotting was estimated by densitometry using the band of each normal tissue as a quantitative control.

mRNA extraction and RT-PCR. mRNAs were isolated from tissues using the RNeasy ${ }^{\circledR}$ Mini kit (Qiagen, Chatsworth, CA, USA), treated with DNase I, and quantified via absorbance at 260 and $280 \mathrm{~nm}$. RT-PCR was conducted using a SuperScript one-step RT-PCR kit (Invitrogen, Carlsbad, CA, USA). Reverse transcription was conducted for $1 \mathrm{~h}$ at $42^{\circ} \mathrm{C}$, and the cDNAs were amplified. The synthesized first-strand cDNA was employed for real-time PCR analysis using SYBR-Green I. The sequences of the specific primers were as follows: PRL-1 forward, 5'-ATGGCTCGAATGAACCGC-3'; PRL-1 reverse, 5'-TGCAACAGTTGTTTCTATGACCG-3'; PRL-2 forward, 5'-ATGAACCGTCCAGCCCCT-3'; PRL-2 reverse, 5'-CTACTGAACACAGCAATGCCATT-3'; PRL-3 forward, 5'-ATGGCTCGGATGAACCGC-3'; PRL-3 reverse, 5'-CTACATAACGCAGCACCGGG-3'; GAPDH forward, 5'-GTCAACGGAATTTGGTCTGTATT-3'; GAPDH reverse, 5'-AGTCTTCTGGGTGGCAGTGAT-3'. Product accumulation was monitored with an iCycler iQ real-time detection system (Bio-Rad Laboratories). The mean cycle threshold value $(\mathrm{Ct})$ was calculated from triplicate samples, and applied to determine the level of gene expression. The sample $\Delta \mathrm{Ct}$ $(\mathrm{S} \Delta \mathrm{Ct})$ values were normalized as the difference between the GAPDH Ct and sample $\mathrm{Ct}$ values.

Cell culture. A549 human lung adenocarcinoma epithelial cell line was grown in DMEM medium (Invitrogen) supplemented with $10 \%(\mathrm{v} / \mathrm{v})$ fetal bovine serum (Hyclone, Logan, UT, USA), $100 \mathrm{IU} / \mathrm{ml}$ of penicillin and $100 \mu \mathrm{g} / \mathrm{ml}$ of streptomycin (Invitrogen). Cells were maintained at $37^{\circ} \mathrm{C}$ in a humidified incubator under $5 \%$ of $\mathrm{CO}_{2}$.

Luciferase reporter assay. For the luciferase reporter assay, p21-Luc (17) and AP-1-Luc reporter plasmid with or without PRL-1, PRL-2 and PRL-3 expression plasmid (Flag-PRL-1, Flag-PRL-2, Flag-PRL-3; Flag-PRL-2 was kindly provided from Dr Sung Hyun Kang and Dr Kwang Hee Bae at Korea Research Institute of Bioscience and Biotechnology) was transfected into cells using Lipofectamine Plus (Invitrogen) in accordance with the supplier's instructions. One microgram of DNA mixed in all samples with $100 \mathrm{ng}$ of CMV-Ren plasmid (Promega, Madison, WI, USA) as an internal control was co-transfected into cells in 6-well plates (Corning Inc., Corning, NY, USA). Luciferase assays were conducted $48 \mathrm{~h}$ after transfection using a Luciferase Assay Reagent kit (Promega) and a Lumat LB9507 luminometer (Berthold, Bad Wildbad, Germany). The values were normalized with Renilla luciferase activity.

WST-1 assay. DNAs (Mock, FLAG-PRL-1, -2 or -3) were transfected into NIH/3T3 cells using Lipofectamine Plus (Gibco-BRL, CA, USA) in accordance with the supplier's instructions in 6-well plates (Corning Inc.). Cell proliferation assay was conducted 3 days after transfection using WST-1 assay kit (Roche). Medium was replaced with $1 \mathrm{ml}$ culture medium and $100 \mu \mathrm{l}$ of the WST-1 reagent was added to each well. The cells were incubated for an additional $2 \mathrm{~h}$ at $37^{\circ} \mathrm{C}$, and absorbance was measured at a wavelength of $450 \mathrm{~nm}$ with a reference wavelength of $630 \mathrm{~nm}$ using a microplate reader (Bio-Rad).

Statistical analysis. Data were analyzed using GraphPad Prism 5.01 for Windows (GraphPad Software for Science Inc., San Diego, CA, USA). Results are presented as means \pm SD. The significance of differences between samples was determined by non-parametric unpaired, two-tailed t-tests.

\section{Results}

Increased expression of $P R L-2$ protein in lung cancer. Patient demography is shown in Table I. Mean age of the patients is 66 
Table I. Demography of lung cancer patients.

\begin{tabular}{lrc}
\hline & \multicolumn{2}{c}{ Patients } \\
\cline { 2 - 3 } Characteristics & Number (N) & Percent (\%) \\
\hline Gender & 25 & 83.3 \\
$\quad$ Male & 5 & 16.7 \\
$\quad$ Female & & \\
Smoking status & 14 & 46.7 \\
$\quad$ Smoker & 16 & 53.3 \\
$\quad$ Non-smoker & & \\
Tumor type & 10 & 33.3 \\
$\quad$ Adenocarcinoma & 19 & 63.3 \\
$\quad$ Squamous cell carcinoma & 1 & 3.4 \\
$\quad$ Pleomorphic carcinoma & & \\
Stage & 16 & 53.3 \\
I & 6 & 20.0 \\
II & 8 & 26.7 \\
III & & \\
\hline
\end{tabular}

( \pm 9$)$ and eight of them are stage III and they are all pathologically confirmed positive N2 nodes after surgery. It has been reported that PRLs especially PRL-3 expression was positively correlated with the advanced stage of lung cancer (11). Thus, we examined protein expression of other PRLs by Western blot analysis. For quantification, we used the ratio of cancer to normal tissues with band intensity which was calculated with densitometry (Table II). Expressions of PRL-2 were higher in cancer than normal tissues in 22 cases (73.3\%). But, the expression levels of PRL-1 and -3 proteins were generally lower in cancer tissues than those of normal tissues. Collectively, only PRL-2 expression was elevated in cancer tissues. Especially, in all stage III patients, cancer tissues expressed higher levels of PRL-2 than normal tissues, but the expression levels of PRL-1 and -3 were not increased in cancerous tissues (Fig. 1A). In addition, the expression of PRL-2 was increased in cancer tissues regardless of stages and those of PRL-1 and -3 were not induced (Fig. 1B-D). Furthermore, the ratio of cancer to normal density of PRL-2 protein was the highest in stage III patients (average values are 2.1 for stage I, 1.88 for stage II and 5.55 for stage III). Therefore, those data indicate that PRL-2 is highly expressed and may play an important role in lung cancers.

Next, to determine transcription of PRLs in lung cancers, we collected and prepared the samples and performed realtime RT-PCR. We compared the ratio of cancer to normal tissues of mRNA of PRLs and all three PRL mRNAs were not significantly increased in cancer tissues (Fig. 1E). Thus, this result indicates that expression of PRL mRNA was not elevated in cancer tissues and the mRNA expression levels of PRLs were not statistically correlated with the stages or other factors of lung cancers.

Decreased p21 transcription activity and increased AP-1 activity by PRLs. To determine functions of PRLs in primary
Table II. Relative expression of PRL-1, -2 and -3 protein in lung cancer tissue.

\begin{tabular}{|c|c|c|c|}
\hline Stage & $\begin{array}{l}\text { PRL-1 } \\
\text { fold }\end{array}$ & $\begin{array}{l}\text { PRL-2 } \\
\text { fold }\end{array}$ & $\begin{array}{l}\text { PRL-3 } \\
\text { fold }\end{array}$ \\
\hline $1 \mathrm{a}$ & 0.13 & 1.67 & 0.45 \\
\hline $1 \mathrm{a}$ & 0.96 & 1.60 & 1.09 \\
\hline $1 \mathrm{a}$ & ND & ND & 1.14 \\
\hline $1 \mathrm{a}$ & 0.86 & 0.81 & 1.20 \\
\hline $1 b$ & ND & 0.13 & 0.39 \\
\hline $1 b$ & 0.64 & 3.94 & 0.80 \\
\hline $1 b$ & 0.63 & 8.94 & ND \\
\hline $1 b$ & ND & 3.50 & 0.55 \\
\hline $1 b$ & 0.19 & 1.39 & 0.01 \\
\hline $1 b$ & 0.43 & 0.45 & 0.76 \\
\hline $1 b$ & 1.98 & 0.61 & 0.74 \\
\hline $1 b$ & ND & ND & 1.67 \\
\hline $1 b$ & ND & 3.43 & 1.25 \\
\hline $1 b$ & 2.06 & 2.17 & 0.76 \\
\hline $1 b$ & 1.04 & 1.99 & 0.45 \\
\hline $1 b$ & 0.57 & 2.92 & 1.06 \\
\hline $2 a$ & 0.28 & 2.88 & ND \\
\hline $2 \mathrm{a}$ & 1.42 & 0.42 & ND \\
\hline $2 \mathrm{a}$ & 1.07 & 3.22 & ND \\
\hline $2 a$ & ND & 1.55 & 1.03 \\
\hline $2 \mathrm{a}$ & 1.62 & 2.15 & 0.56 \\
\hline $2 \mathrm{a}$ & 0.07 & 1.08 & ND \\
\hline $3 a$ & ND & 13.71 & ND \\
\hline $3 a$ & 0.14 & 4.21 & 0.12 \\
\hline $3 a$ & 0.22 & 11.00 & ND \\
\hline $3 a$ & 0.19 & 1.04 & ND \\
\hline $3 a$ & ND & 1.70 & 0.23 \\
\hline $3 a$ & 1.04 & 4.75 & 1.34 \\
\hline $3 b$ & 0.07 & 0.96 & 0.06 \\
\hline $3 b$ & 2.97 & 7.00 & 1.39 \\
\hline $\begin{array}{l}\text { No (cancer/ } \\
\text { normal >1) }\end{array}$ & 8 & 22 & 9 \\
\hline Means $\pm S D$ & $0.84 \pm 0.77$ & $3.19 \pm 3.28$ & $0.78 \pm 0.47$ \\
\hline
\end{tabular}

Band intensity of Western blotting was estimated by densitometry using the band of each normal tissue as the quantitative control. The ratio of lung cancer and normal tissue (expressed as fold) are shown here (ND, not detectable).

lung cancer cells, we first performed p21-luciferase reporter assay, since it is known that PRLs regulate p21 (15). Thus, we performed reporter assay to investigate the effect of PRLs on the activity of p21 promoter using overexpression of PRLs in A549 human lung adenocarcinoma epithelial cell line. The activity of p21 promoter was down-regulated by all PRLs (Fig. 2A). Thus, this result indicates that PRLs negatively regulate the activity of $\mathrm{p} 21$ and may be involved in the tumor progression by increasing the cell cycle. 


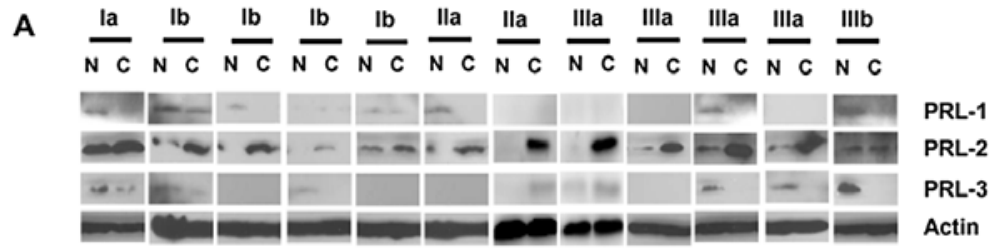

B

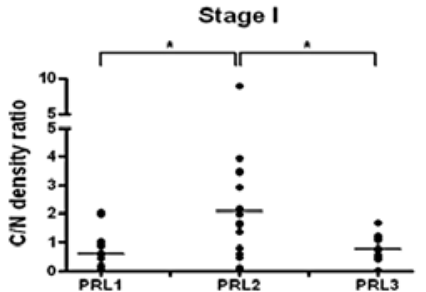

D

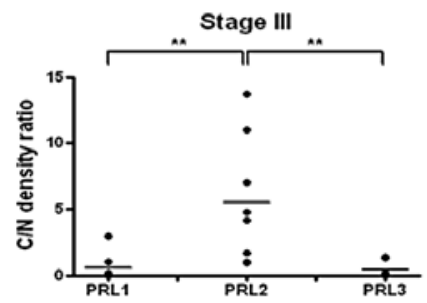

C

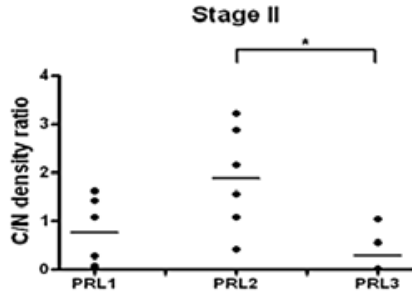

E

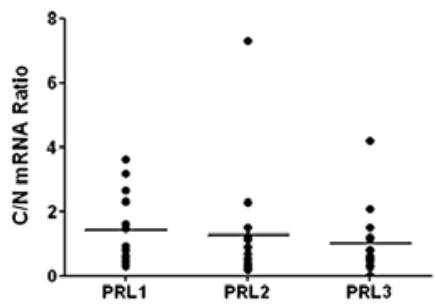

Figure 1. Expression levels of PRL-1, -2 and -3 proteins and mRNAs in lung cancer tissue. (A) PRL-2 was highly expressed in lung cancer (C) compared with normal tissue (N) but PRL-1 and -3 were hardly detected in cancer tissues. Representative data from patients of stage I, II, III are shown, and $\beta$-actin was used as a loading control. Density ratios of cancer to normal tissues of PRL-1, -2 and -3 were calculated from different stages of patients [(B) stage I, (C) stage II and (D) stage III]. Regardless of stages, PRL-2 ratio was significantly increased ( $\left.{ }^{*} \mathrm{p}<0.05,{ }^{* *} \mathrm{p}<0.005\right)$. (E) The transcription levels of PRLs were analyzed by real-time RT-PCR using specific primers of PRL-1, -2 and -3. GAPDH was used as house keeping control to normalize the relative amount of PRLs. Ratios of cancer to normal tissues of PRL-1, -2 and -3 mRNA levels were calculated.

A

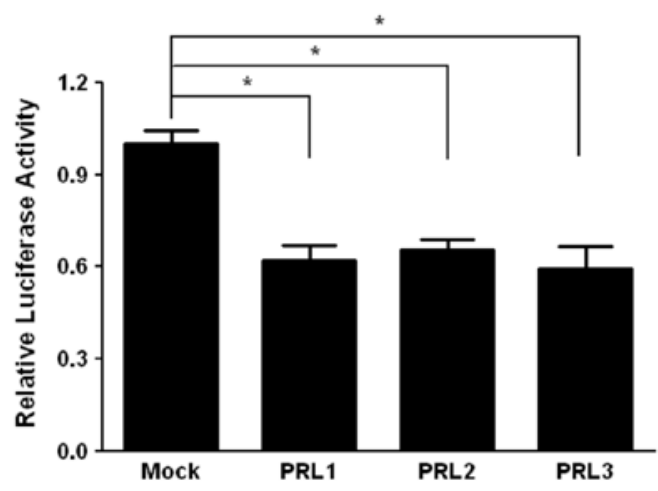

B

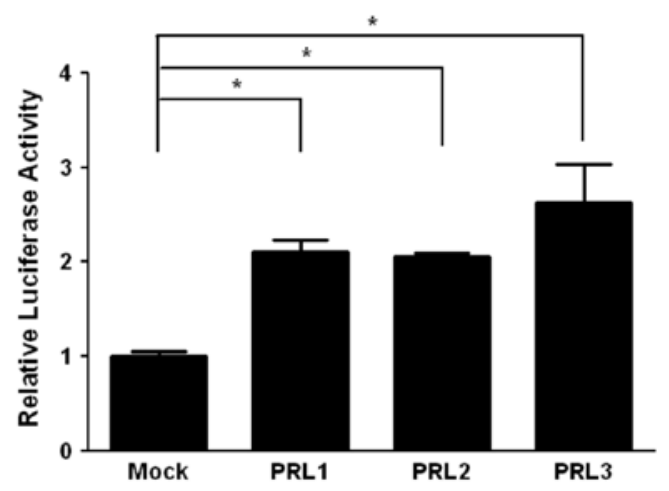

Figure 2. Reduced p21 promoter activity and increased AP-1 activity by PRLs. Luciferase assays were conducted 48 h after transfection using a Luciferase Assay Reagent kit and a Lumat LB9507 luminometer. The relative luciferase activity was calculated by dividing each normalized average luciferase value by the normalized average mock luciferase value. (A) Promoter activity of p21 was numerated by relative luciferase activity. (B) AP-1 activity was proportional to the relative luciferase activity. Data represent means $\pm \mathrm{SD}\left({ }^{*} \mathrm{p}<0.05\right)$.

Next, we investigated the possibility of regulation of AP-1 by PRLs because it has been shown that PRL-2 regulates MAP kinase pathway (18). We performed additional AP-1-luciferase reporter assay through overexpression of PRLs in A549 cells. Opposite to the p21 reporter result, the activity of AP-1 was induced by all PRLs (Fig. 2B). Therefore, this result suggests that PRLs positively regulate AP-1 activity and could drive tumor progression through AP-1-dependent signaling.
Enhanced cell proliferation with PRL overexpression. Lastly, we examined the effect of PRLs on cell proliferation by introducing PRLs into NIH/3T3 cells. Transfection of all PRLs showed significant increased cell proliferation and PRL-2 seemed to be potent (Fig. 3). Therefore, the results indicate that PRLs induce cell proliferation, and may have functional roles in lung cancers by increasing cell proliferation through negative regulation of $\mathrm{p} 21$ and positive regulation of AP-1 pathway. 


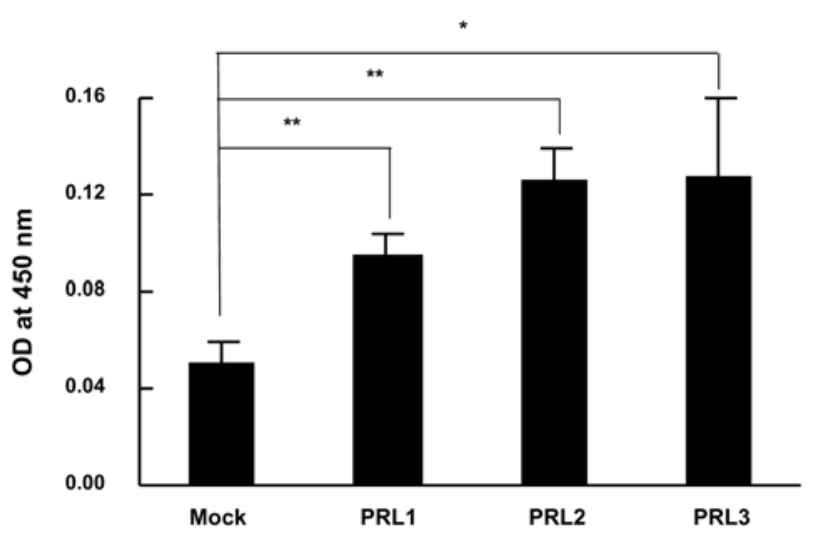

Figure 3. Increased proliferation by transfection of PRLs. Cell proliferation assay was conducted 3 days after transfection using a WST-1 assay kit. Compared to Mock transfection, all PRL transfected cells showed increased proliferation of NIH/3T3 cells. Especially, PRL-2 tranfected cell showed relatively higher proliferation. The data are expressed as the means $\pm \mathrm{SD}\left({ }^{*} \mathrm{p}<0.05\right.$, *** $\mathrm{p}<0.005)$

\section{Discussion}

PRLs are highly expressed in various cancers, especially in metastatic cancers (2). Thus, PRL family has been implicated as a candidate for a biomarker in metastatic and advanced cancers. In this study, we compared relative expressions of three PRLs in lung cancers and distal normal tissues from the same lobes. Our results showed that expression of PRL-2 protein in lung cancers was higher than that in normal tissues whereas expression of mRNA was not enhanced indicating that there is no correlation between mRNA and protein expression. In contrast, PRL-1 and -3 protein expressions in primary lung cancer were lower than normal tissues. We also observed that expression level of PRL-2 protein was relatively more abundant compared to that of PRL-1 or PRL-3 in cancer tissues. Co-transfection of PRLs with p21 promoter-reporter showed that all three PRLs suppressed the activity of p21 promoter indicating PRLs can be negative regulators of the tumor suppressor gene in lung cancer. Furthermore, co-transfection of PRLs enhanced AP-1 activity suggesting PRLs can be positive regulators of the AP-1-dependent signaling. Lastly, overexpression of PRLs induced increased cell proliferation. Therefore, the results suggest that among three PRLs, PRL-2 plays a critical role in the pathogenesis of lung cancer and could be a biomarker of this cancer by substituting for the function of other PRLs.

Among PRLs, PRL-1 and -2 are widely expressed in human tissues. However, PRL-3 is primarily expressed in heart and skeletal muscle, although low level expression can be detected in other tissues as well $(2,19)$. So it has been thought that they function in basic processes common to many tissues and cell types (20). Many tissues that do not express or express only low levels of PRL-3 except heart and skeletal muscle are unable to cope with elevated levels of its activity and thus are susceptible to the tumorigenic effects of PRL-3 (2). For this reason, many studies have focused on PRL-3 in lung cancer. The Ming group has reported that PRL-3 is expressed in all 94 cases of primary lung cancer patients and highly expressed in
64 cases $(68 \%)$ by in situ immunohistochemical staining (11). In addition, the expression level is significantly associated with advanced clinical stage, lymph node and distant metastasis. The survival rate is also significantly associated with the level of PRL-3 expression. However, the group of Yamashita has shown that the levels of PRL-3 gene expression in primary lesions are slightly decreased compared with those in normal tissue in metastatic lung cancers (10). Further, those in metastatic lesions are extremely down-regulated in the synchronous cases at 10 times less than that of the normal tissue. Because of this discrepancy, we analyzed expression patterns of PRLs in primary lung cancer patients and showed that PRL-2 is a major PRL expressed in lung cancer tissues. Furthermore, recent study showed that PRL-2 plays an important role in breast cancer progression (18). However, the expression of PRL-2 protein was not correlated with that of mRNA expression, thus, the suppression of mRNA translation by PCBP1 like PRL-3 (16) or post-translational modification for stability of the protein might be important.

Human PRL-1 and -2 share the closest amino acid identity at $87 \%$. PRL-1 and -3 exhibit $79 \%$ sequence identity, and PRL-2 and -3 have $76 \%$ sequence identity (21). PRLs are highly preserved in mammalians and there is also high level of homology among mammalian PRLs. The amino acid sequences for human and mouse PRL-1 and -2 are identical, whereas PRL-3 is $96 \%$ identical between two species (22). Thus, it is possible that all three PRLs have functional redundancy. To investigate the functions of PRL-2 in lung cancer, we first performed p21-promoter reporter assay because it is well known that PRLs induce cell proliferation and malignant transformation through down-regulation of p21 (15). In p21-promoter luciferase reporter assay, all PRLs decreased the activity of p21 promoter. Thus, our data suggest that PRL-2 can substitute the function of other PRL family in primary lung cancer unlike other cancers.

PRLs play a profound role in the development and metastasis of various types of cancers. Those effects are mediated through several signaling pathways such as PI3K/Akt, Rho GTPase, cyclin-dependent kinase 2 (CDK2), angiotensin II and p53 signaling $(9,12-15,19,23)$. The PI3K/Akt is an important signaling pathway in cell survival, and it is frequently activated in human cancers. We have recently reported that PRL-1 is a new p53 target and down-regulates the p53 protein via Akt and Rho signaling (14). In another study, PRL-3 promotes VEGF expression by increasing phosphorylation of Erk and up-regulating the levels and activities of Rho-A and Rho-C (11). On the other hand, Jak/Stat pathways are activated in response to cytokines and growth factors. Recently it has been reported that phosphorylated Stat 3 was frequently observed in the tumor derived cell lines and tumor specimens from human cancers (24). Because PRLs are involved in various intracellular signaling pathways, we examined the activity of AP-1, one of the well-known cell growth related pathway, with co-transfection of PRLs and AP-1 reporter. In the reporter assay, our data showed that overexpression of PRLs increased the activity of AP-1, thus, activating cell growth signaling. Finally, we examined the effect of PRLs on cell proliferation because PRLs especially PRL-2 can suppress p21 and activate AP-1. Consistent with our results, overexpression of PRLs into NIH/3T3 cells can induce increased cell proliferation. Thus, 
those results indicate that PRLs positively regulate AP-1dependent signaling pathways, and have functional roles in lung cancers by increasing cell proliferation.

In conclusion, our study has shown that the expression levels of PRL-1 and -3 were decreased in lung cancer, in contrast, that of PRL-2 was increased in primary lung cancer. Overexpression of all three PRLs suppressed the transcription of p21, a tumor suppressor. Furthermore, AP-1 activity was enhanced by overexpression of PRLs and lastly, transfection of PRLs can induce increased cell proliferation. Therefore, these results suggest that PRL-2 plays an important role in lung cancer and could be a biomarker of lung cancer.

\section{Acknowledgements}

This study was supported by a grant of the Korea Healthcare Technology R\&D Project, Ministry for Health, Welfare and Family Affairs, Republic of Korea (A084832, to S.-H.L.).

\section{References}

1. Stephens B, Han H, Gokhale V and von Hoff D: PRL phosphatases as potential molecular targets in cancer. Mol Cancer Ther 4: 1653-1661, 2005.

2. Bessette D, Qiu D and Pallen C: PRL PTPs: mediators and markers of cancer progression. Cancer Metastasis Rev 27: 231-252, 2008

3. Achiwa $\mathrm{H}$ and Lazo J: PRL-1 tyrosine phosphatase regulates c-Src levels, adherence, and invasion in human lung cancer cells. Cancer Res 67: 643-650, 2007.

4. Saha S, Bardelli A, Buckhaults P, et al: A phosphatase associated with metastasis of colorectal cancer. Science 294: 1343-1346, 2001.

5. Wang Q, Holmes D, Powell S, Lu Q and Waxman J: Analysis of stromal-epithelial interactions in prostate cancer identifies PTPCAAX2 as a potential oncogene. Cancer Lett 175: 63-69, 2002.

6. Parker B, Argani P, Cook B, et al: Alterations in vascular gene expression in invasive breast carcinoma. Cancer Res 64: 7857-7866, 2004.

7. Miskad U, Semba S, Kato H and Yokozaki H: Expression of PRL-3 phosphatase in human gastric carcinomas: close correlation with invasion and metastasis. Pathobiology 71: 176-184, 2004.

8. Wu X, Zeng $\mathrm{H}$, Zhang X, et al: Phosphatase of regenerating liver-3 promotes motility and metastasis of mouse melanoma cells. Am J Pathol 164: 2039-2054, 2004.

9. Wang H, Quah S, Dong J, Manser E, Tang J and Zeng Q: PRL-3 down-regulates PTEN expression and signals through PI3K to promote epithelial-mesenchymal transition. Cancer Res 67 2922-2926, 2007.
10. Yamashita S, Masuda Y, Matsumoto K, et al: Down-regulation of the human PRL-3 gene is associated with the metastasis of primary non-small cell lung cancer. Ann Thorac Cardiovase Surg 13: 236-239, 2007.

11. Ming J, Liu N, Gu Y, Qiu X and Wang E: PRL-3 facilitates angiogenesis and metastasis by increasing ERK phosphorylation and up-regulating the levels and activities of Rho-A/C in lung cancer. Pathology 41: 118-126, 2009.

12. Liang F, Liang J, Wang W, Sun J, Udho E and Zhang Z: PRL3 promotes cell invasion and proliferation by down-regulation of Csk leading to Src activation. J Biol Chem 282: 5413-5419, 2007.

13. Fiordalisi J, Keller P and Cox A: PRL tyrosine phosphatases regulate rho family GTPases to promote invasion and motility. Cancer Res 66: 3153-3161, 2006.

14. Min S, Kim D, Heo Y, et al: New p53 target, phosphatase of regenerating liver 1 (PRL-1) down-regulates p53. Oncogene 28: $545-554,2009$.

15. Werner S, Lee P, DeCamp M, Crowell D, Randall S and Crowell P: Enhanced cell cycle progression and down-regulation of p21(Cip1/Waf1) by PRL tyrosine phosphatases. Cancer Lett 202: 201-211, 2003.

16. Wang H, Vardy LA, Tan CP, et al: PCBP1 suppresses the translation of metastasis-associated PRL-3 phosphatase. Cancer Cell 18: 52-62, 2010.

17. Resnick-Silverman L, St Clair S, Maurer M, Zhao K and Manfredi J: Identification of a novel class of genomic DNA-binding sites suggests a mechanism for selectivity in target gene activation by the tumor suppressor protein p53. Genes Dev 12: 2102-2107, 1998.

18. Hardy S, Wong NN, Muller WJ, Park M and Tremblay ML: Overexpression of the protein tyrosine phosphatase PRL-2 correlates with breast tumor formation and progression. Cancer Res 70: 8959-8967, 2010.

19. Matter W, Estridge T, Zhang C, et al: Role of PRL-3, a human muscle-specific tyrosine phosphatase, in angiotensin-II signaling. Biochem Biophys Res Commun 283: 1061-1068, 2001.

20. Dumaual C, Sandusky G, Crowell P and Randall S: Cellular localization of PRL-1 and PRL-2 gene expression in normal adult human tissues. J Histochem Cytochem 54: 1401-1412, 2006.

21. Liu Y, Li H, Lou X and Lei J: Expression of phosphatase of regenerating liver 1 and 3 mRNA in esophageal squamous cell carcinoma. Arch Pathol Lab Med 132: 1307-1312, 2008.

22. Zhao W and Wang X: Phosphatases of regenerating liver: a novel target in human solid tumors. Chin Med J (Engl) 121: 1469-1474, 2008.

23. Min S, Kim D, Heo Y, Kim H, Kim I and Yoo O: Down-regulation of p53 by phosphatase of regenerating liver 3 is mediated by MDM2 and PIRH2. Life Sci 86: 66-72, 2010.

24. Lin J, Jin X, Rothman K, Lin H, Tang H and Burke W: Modulation of signal transducer and activator of transcription 3 activities by $\mathrm{p} 53$ tumor suppressor in breast cancer cells. Cancer Res 62: 376-380, 2002. 